\title{
ARL Estimation of the Control Chart of Log Likelihood Ratios' Sum for Markov Sequence
}

\author{
Yi Guo, ${ }^{1}$ Lei Gao, ${ }^{1}$ and Yan Zhu ${ }^{2}{ }^{2}$ \\ ${ }^{1}$ Department of Automation, Shanghai Jiao Tong University, Shanghai 200240, China \\ ${ }^{2}$ College of Science, University of Shanghai for Science and Technology, Shanghai 200093, China
}

Correspondence should be addressed to Yan Zhu; zhuyan@usst.edu.cn

Received 10 December 2020; Revised 22 April 2021; Accepted 28 April 2021; Published 10 May 2021

Academic Editor: Niansheng Tang

Copyright ( 2021 Yi Guo et al. This is an open access article distributed under the Creative Commons Attribution License, which permits unrestricted use, distribution, and reproduction in any medium, provided the original work is properly cited.

To evaluate the surveillance performance of a control chart with the charting statistic of the sum of log likelihood ratios in the statistical process control (SPC), in this paper, we give the proof procedure based on Markov chains for the asymptotic estimation of the average run length (ARL) for this kind of chart. The out-of-control $A_{R L}$ is approximately equal to 1 for any fixed in-control $\mathrm{ARL}_{0}$ with a negative control limit. By the equivalence between limit distribution of a sum and that of a suprema sum of Markov chain, we derive the estimation of $\mathrm{ARL}_{1}$ with a large enough positive control limit. Numerical experiments are conducted to confirm our results.

\section{Introduction}

The main aim of SPC is to detect an abrupt change in the observation of time series as soon as possible after the change has happened. The study, found in [1], is the first to show the design of the control chart for quickly detecting possible changes in the underlying process. Subsequently, a great number of control charts have been proposed and come into a wider use in many fields, such as environmental control $[2,3]$, biostatistics $[4,5]$, clinical medicine [6,7], economics and finance [8], industrial quality and process control $[9,10]$, process monitoring [11-16], public health $[7,14,16]$, and social network [17].

Obviously, the research on constructing various control charts for surveillance has never stopped since Shewhart presented this method. The existing theoretical studies in statistical monitoring, on the whole, can be classified into three areas. The first category is the optimal economic or economic-statistical design, where, in most cases, the net sum of all costs is minimized [18-21]. The ideas of the second category are the choice of the statistical parameters of a control chart to minimize the out-of-control $\mathrm{ARL}_{1}$ for a given in-control $\mathrm{ARL}_{0}$ or a probability of false alarm, and it is named as the optimal statistical design [16, 22-25]. Recently, the theoretical approximation to ARL was proposed in [26]. Detecting changes in distribution of the optimal control charts is the last category, which can be regarded as a kind of optimal stopping time [27-29]. This metric for defining optimality is based on that it has smallest out-ofcontrol $\mathrm{ARL}_{1}$ among all control charts with either a given probability of a false alarm no greater than a preset level, or a given false alarm rate no less than a given value. So, this more mathematical area is thus related to statistical design. In the following argument, we will focus on the third type of these bibliographies mentioned above.

Consider the Gaussian observation sequence $\left\{X_{0}, X_{1}, X_{2}, \ldots\right\}$, whose distribution may change at time $\tau(\geq 1)$. Let $p_{0}\left(x_{0}, x_{1}, x_{2}, \ldots, x_{n}\right)(n<\tau) \quad$ and $p_{\tau}\left(x_{\tau}, x_{\tau+1}, \ldots\right)(n \geq \tau)$ be the prechange probability density function and the postchange probability density function of $\left\{X_{0}, X_{1}, X_{2}, \ldots\right\}$, respectively. Denote the postchange joint probability distribution, expectation, and variance by $\mathbb{P}_{\tau}, \mathbb{E}_{\tau}$, and $\mathbb{D}_{\tau}$, respectively. Especially, when the change time $\tau=\infty$, we suppose that a change never occurs. Frisén 
[30] showed that there exists a positive value $c$ such that the control chart with the charting statistic of the sum of log likelihood ratios (SLR),

$$
T_{\text {SLR }}(c)=\min \left\{n: \sum_{k=1}^{n}\left(X_{k}-\frac{\mu}{2}\right) \geq c, n \geq 1\right\},
$$

is optimal in the sense,

$$
\operatorname{Inf}_{T: \mathbb{P}_{0}(T \geq \tau) \geq 1-\alpha} \mathbb{E}_{1}(T)=\mathbb{E}_{1}\left(T_{\text {SLR }}(c)\right),
$$

when the change is the case of a shift in the mean of $\left\{X_{0}, X_{1}, X_{2}, \ldots\right\}$, where $T$ denotes the control chart test, $\tau=$ 1 is the change-point time, and $c>0$ is the constant control limit such that $\mathbb{P}_{0}\left(T_{\mathrm{SLR}}(c) \geq \tau\right)=1-\alpha>0$. Although the optimality of $T_{\text {SLR }}$ has been proved in [30], there were few surveys and bibliographies which considered the performance of the control chart $T_{\text {SLR }}$ for monitoring the change point in the process.

Therefore, in this paper, we regard the ARL as the criterion of optimality of a control chart. This is because the ARL is the connection among itself, control limit, and statistical properties of the observation sequence $\left\{X_{0}, X_{1}, X_{2}, \ldots\right\}$. In Section 2, we prove that the out-ofcontrol $A R L_{1}$ is approximately equal to 1 for a given $A R L_{0}$ as the control limit $c$ is negative. In Section 3, we apply the equivalence between limit distribution of a sum and that of a suprema sum of Markov chain to obtain the estimation of $\mathrm{ARL}_{1}$ when the control limit is some large enough positive constant. Finally, we conduct some numerical experiments to verify our theoretical analysis in Section 4.

\section{Estimation of $A R L_{1}$ with a Negative Control Limit}

In this section, we consider a constant control limit $\exp (c)$ to construct a control chart $T_{Z}(c)$ and estimate the corresponding in-control $\mathrm{ARL}_{0}$ and out-of-control $\mathrm{ARL}_{1}$. Let the observations $\left\{X\left(t_{0}\right), X\left(t_{1}\right), X\left(t_{2}\right), \ldots\right\}$ be a time-homogeneous Markov chain with the discrete state space $S=\left\{s_{1}, s_{2}, \ldots\right\}$. Here, we consider only detecting the change at the initial time $\tau=1$, and the Markov chain $\left\{X\left(t_{k}\right), k \geq 0\right\}$ is positive recurrence with the prechange transition probability $p_{0}(y \mid x)$ and the postchange transition probability $p_{1}(y \mid x)$. For convenience, we use $X_{k}$ instead of $X\left(t_{k}\right)$ for $k \geq 0$. Similar to the notations defined in Section 1 , we use $\mathbb{P}_{0}, \mathbb{E}_{0}$, and $\mathbb{D}_{0}$ to denote, respectively, the prechange joint probability distribution, expectation, and variance of $\left\{X_{0}, X_{1}, X_{2}, \ldots\right\}$.

For ease of the subsequent analysis processing, we choose a large enough positive number $N$ to cut out the first $N+1$ terms of the sequence $\left\{X_{0}, X_{1}, X_{2}, \ldots\right\}$. In other words, the sequence $\left\{X_{0}, X_{1}, X_{2}, \ldots, X_{N}\right\}$ tends to $\left\{X_{0}, X_{1}, X_{2}, \ldots\right\}$ when $N$ goes to infinity. The optimality of the control chart $T_{Z}(c)$ for the Markov chain
$\left\{X_{0}, X_{1}, X_{2}, \ldots, X_{N}\right\}$ appeared in [31]. Now, we define the charting statistics as

$$
Y_{k}=\prod_{j=1}^{k} \frac{p_{1}\left(X_{j} \mid X_{j-1}\right)}{p_{0}\left(X_{j} \mid X_{j-1}\right)}, \quad 1 \leq k \leq N .
$$

Let $Z_{j}=\ln \left\{p_{1}\left(X_{j} \mid X_{j-1}\right) / p_{0}\left(X_{j} \mid X_{j-1}\right)\right\}$ for $1 \leq j \leq N$; then, $\ln Y_{k}=\sum_{j=1}^{k} Z_{j}$ for $1 \leq k \leq N$. For any state $s_{i}$, let $\tau_{k}^{i}$ be the time of $k$ th visit of state $s_{i}$ and $N_{n}^{i}$ be the number of times for visiting $s_{i}$ from time 0 to time $n$. The cumulative sums of $Z_{k}$ and $Z_{k}-\mathbb{E}_{1} Z_{k}$ on the $k$ th block of time are, respectively, defined by

$$
\eta_{k}^{i}=\sum_{l=\tau_{k-1}^{i}+1}^{\tau_{k}^{i}} Z_{l}, \widetilde{\eta}_{k}^{i}=\sum_{l=\tau_{k-1}^{i}+1}^{\tau_{k}^{i}}\left(Z_{l}-\mathbb{E}_{1} Z_{l}\right),
$$

for $k=1,2, \ldots, N_{n}^{i}$ and $\tau_{0}^{1}=0$. Then, both $\eta_{1}, \ldots, \eta_{N_{n}^{i}}$ and $\tilde{\eta}_{1}, \ldots, \widetilde{\eta}_{N^{i}}$ are sequences of independent and identically distributed (i.i.d.) random variables.

Throughout this paper, we assume that

(i) $\mathbb{P}_{0}\left(s_{j} \mid s_{i}\right)=0$ if and only if $\mathbb{P}_{1}\left(s_{j} \mid s_{i}\right)=0$, as well as $\mathbb{P}_{1}\left(s_{j} \mid s_{i}\right) / \mathbb{P}_{0}\left(s_{j} \mid s_{i}\right)$ has no atom with respect to $\mathbb{P}_{0}$ for $s_{i}, s_{j} \in S$. Here, we define $0 / 0=1$.

(ii) $\sup _{s_{i} \in S} \mathbb{E}_{0}\left(Z_{1}^{2} \mid X_{0}=s_{i}\right)<+\infty$.

The sum of $Z_{1}, \ldots, Z_{N}$ is written by $S_{N}=\sum_{k=1}^{N} Z_{k}$. Set $\mu_{i, j}=\mathbb{E}_{j}\left(\eta_{1}^{i}\right) / \mathbb{E} \tau_{1}^{i}, \quad \tilde{\mu}_{i, j}=\mathbb{E}_{j}\left|\widetilde{\eta}_{1}^{i}\right| / \mathbb{E} \tau_{1}^{i}, \quad$ and $\sigma_{i, j}=\mathbb{E}_{j}\left(\eta_{1}^{i}-\mathbb{E}_{j} \eta_{1}^{i}\right)^{2} / \mathbb{E} \tau_{1}^{i}$ and $\widetilde{\sigma}_{i, 1}=\mathbb{E}_{1}\left(\left|\widetilde{\eta}_{1}^{i}\right|-\mathbb{E}_{1}\left|\tilde{\eta}_{1}^{i}\right|\right)^{2} / \mathbb{E} \tau_{1}^{i}$. Moreover, for $j=0,1$, let

$$
\begin{aligned}
& \underline{\mu}_{j}=\min _{i \in\{1,2, \ldots\}} \mu_{i, j}, \\
& \bar{\mu}_{j}=\max _{i \in\{1,2, \ldots\}} \mu_{i, j}, \\
& \underline{\sigma}_{j}=\min _{i \in\{1,2, \ldots\}} \sigma_{i, j}, \\
& \bar{\sigma}_{j}=\max _{i \in\{1,2, \ldots\}} \sigma_{i, j},
\end{aligned}
$$$$
\overline{\widetilde{\mu}}_{1}=\max _{i \in\{1,2, \ldots\}} \tilde{\mu}_{i, 1}, \quad \overline{\widetilde{\sigma}}_{1}=\max _{i \in\{1,2, \ldots\}} \tilde{\sigma}_{i, 1} .
$$

Then, for large $N$, we have

$$
\begin{aligned}
& \mathbb{E}_{j}\left(\sum_{k=1}^{N_{n}^{i}} \eta_{k}^{i}\right)=n \mu_{i, j}(1+o(1)), \\
& \mathbb{D}_{j}\left(\sum_{k=1}^{N_{n}^{i}} \eta_{k}^{i}\right)=n \sigma_{i, j}^{2}(1+o(1)) .
\end{aligned}
$$

Now, we define a control chart $T_{Z}(c)$ in the following with the charting statistics of the sum of log likelihood ratios for detecting the change in distribution of the Markov sequence $X_{0}, X_{1}, \ldots, X_{N}$ : 


$$
T_{Z}(c)=\min \left\{n: Y_{n} \geq \exp (c), 1 \leq n \leq N\right\}=\min \left\{n: \sum_{k=1}^{n} Z_{k} \geq c, 1 \leq n \leq N\right\},
$$

for some constant $c$.

Now, we are ready to estimate $\mathrm{ARL}_{1}$. Based on Section 3 presented in [30], $\mathrm{ARL}_{0}$ is the average run length until an alarm is signaled, so $\mathrm{ARL}_{0}$ and $\mathbb{E}_{0}\left(T_{Z}(c)\right)$ are equivalent. Consequently, we use the estimator of $\mathbb{E}_{0}\left(T_{Z}(c)\right)$ to substitute $\mathrm{ARL}_{0}$ in the subsequent analysis. The main result is proposed in the following theorem.

Theorem 1. Let $\left\{X_{k}, k \geq 0\right\}$ be a time-homogeneous Markov chain satisfying conditions (i) and (ii). Then, for a given $A R L_{0}=L$ and $1<A R L_{0}<N+1$, there exists a negative number $c^{*}=c^{*}(N, L)$ such that

$$
\begin{aligned}
& \operatorname{ARL}_{0}\left(T_{Z}\left(c^{*}\right)\right)=\mathbb{E}_{0}\left(T_{Z}\left(c^{*}\right)\right)=L, \\
& \operatorname{ARL}_{1}\left(T_{Z}\left(c^{*}\right)\right)=\mathbb{E}_{1}\left(T_{Z}\left(c^{*}\right)\right)=1+o(1),
\end{aligned}
$$

as $N \longrightarrow \infty$.

Proof. Choose the control limit $c_{1}=c_{1}(N)=\underline{\mu}_{0} N$ and note that

$$
\begin{aligned}
\mathbb{E}_{0}\left(\eta_{1}^{i} \mid X_{0}=s_{i}\right) & =\mathbb{E}_{0}\left(\sum_{n=1}^{N}\left(I_{\left\{\tau_{1}=n\right\}} \sum_{k=1}^{N} Z_{k}\right) \mid X_{0}=s_{i}\right) \\
& =\mathbb{E}_{0}\left(\sum_{k=1}^{\infty}\left(I_{\left\{\tau_{1} \geq k\right\}} Z_{k}\right) \mid X_{0}=s_{i}\right) \\
& =\sum_{k=1}^{\infty} \mathbb{E}_{0}\left[\zeta\left(X_{0}, \cdots, X_{k-1}\right) Z_{k} \mid X_{0}=s_{i}\right],
\end{aligned}
$$

where $I_{\left\{\tau_{1} \geq k\right\}}=\zeta\left(X_{0}, \ldots, X_{k-1}\right) \in \mathscr{F}_{k-1}$. Let $\mathbb{E}_{0, s_{i}}=\mathbb{E}_{0}[\zeta$ $\left.\left(X_{0}, \ldots, X_{k-1}\right) Z_{k} \mid X_{0}=s_{i}\right]$. Using the law of total expectation and Markov property, we obtain

$$
\begin{aligned}
\mathbb{E}_{0, s_{i}}\left[\zeta\left(X_{0}, \ldots, X_{k-1}\right) Z_{k}\right] & =\mathbb{E}_{0, s_{i}}\left[\mathbb{E}_{0, s_{i}}\left(\zeta\left(X_{0}, \ldots, X_{k-1}\right) Z_{k} \mid \mathscr{F}_{k-1}\right)\right] \\
& =\mathbb{E}_{0, s_{i}}\left[\zeta\left(X_{0}, \ldots, X_{k-1}\right) \mathbb{E}_{0, s_{i}}\left(Z_{k} \mid X_{k-1}\right)\right] .
\end{aligned}
$$

Since $\ln x$ is a concave function, by Jensen's inequality, we have

$$
\begin{aligned}
\mathbb{E}_{0, s_{i}}\left(Z_{k} \mid X_{k-1}\right) & =\mathbb{E}_{0, s_{i}}\left(\ln \frac{p_{1}\left(X_{k} \mid X_{k-1}\right)}{p_{0}\left(X_{k} \mid X_{k-1}\right)} \mid X_{k-1}\right) \\
& \leq \ln \mathbb{E}_{0, s_{i}}\left(\frac{p_{1}\left(X_{k} \mid X_{k-1}\right)}{p_{0}\left(X_{k} \mid X_{k-1}\right)} \mid X_{k-1}\right)=0 .
\end{aligned}
$$

Substituting (13) into (12) and combining (11), we can get that $\mu_{i, 0}<0$ for any $i$. Similarly, we can prove $\mu_{i, 1}>0$ for any $i$. Hence, $\underline{\mu}_{0}, \bar{\mu}_{0}<0$ and $\underline{\mu}_{1}, \bar{\mu}_{1}>0$; then, $\lim _{N \rightarrow \infty} c_{1}(N)=-\infty$. Let $\widetilde{m}=\left[\varepsilon_{1} N\right]$ for some $\varepsilon_{1}<1$, where $[x]$ denotes the integer part of $x$. Then,

$$
\begin{aligned}
\mathbb{E}_{0}\left(T_{Z}\left(c_{1}\right)\right) & =\mathbb{P}_{0}\left(T_{Z}\left(c_{1}\right)>0\right)+\sum_{m=1}^{N} \mathbb{P}_{0}\left(T_{Z}\left(c_{1}\right)>m\right) \\
& =1+\sum_{m=1}^{\tilde{m}-1} \mathbb{P}_{0}\left(T_{Z}\left(c_{1}\right)>m\right)+\sum_{m=\tilde{m}}^{N} \mathbb{P}_{0}\left(T_{Z}\left(c_{1}\right)>m\right) \leq 1+\sum_{m=1}^{\tilde{m}-1} \mathbb{P}_{0}\left(T_{Z}\left(c_{1}\right)>m\right)+(N-\tilde{m}+1) \mathbb{P}_{0}\left(T_{Z}\left(c_{1}\right)>\widetilde{m}\right) .
\end{aligned}
$$

The total probability formula tells us that 


$$
\begin{aligned}
\mathbb{P}_{0}\left(T_{Z}\left(c_{1}\right)>\widetilde{m}\right) & =\mathbb{P}_{0}\left(\max _{1 \leq k \leq \tilde{m}} S_{k} \leq c_{1}\right) \leq \mathbb{P}_{0}\left(S_{\widetilde{m}} \leq c_{1}\right) \\
& =\sum_{i=1}^{\infty} \mathbb{P}_{0}\left(X_{0}=s_{i}\right) \mathbb{P}_{0}\left(s_{\tilde{m}} \leq c_{1} \mid X_{0}=s_{i}\right) .
\end{aligned}
$$

Equations (4) and (7) yield that

$$
\begin{aligned}
& \mathbb{P}_{0}\left(s_{\widetilde{m}} \leq c_{1} \mid X_{0}=s_{i}\right)=\mathbb{P}_{0}\left(\sum_{k=1}^{N_{\widetilde{m}}^{i}} \eta_{k}^{i} \leq c_{1}-\sum_{\substack { k=\tau_{N}^{i}+1 \\
\begin{subarray}{c}{m{ k = \tau _ { N } ^ { i } + 1 \\
\begin{subarray} { c } { m } }\end{subarray}}^{N_{\widetilde{m}}^{i}} Z_{k} \mid X_{0}=s_{i}\right) \\
& =\mathbb{P}_{0}\left(\frac{\sum_{k=1}^{N_{\widetilde{m}}^{i}} \eta_{k}^{i}-\mathbb{E}_{0}\left(\sum_{k=1}^{N_{\tilde{m}}^{i}} \eta_{k}^{i}\right)}{\mathbb{D}_{0}\left(\sum_{k=1}^{N_{\tilde{m}}^{i}} \eta_{k}^{i}\right)} \geq \frac{-c_{1}+\tilde{m} \mu_{i, 0}(1+o(1))}{\sigma_{i, 0} \sqrt{\widetilde{m}}}+\frac{\sum_{k=\tau_{N_{\widetilde{m}}^{i}}+1}^{N_{\widetilde{m}}^{i}} Z_{k}}{\sigma_{i, 0} \sqrt{\widetilde{m}}} \mid X_{0}=s_{i}\right) .
\end{aligned}
$$

Note that $\widetilde{m}=\left[\varepsilon_{1} N\right]$ and $\sum_{k=\tau^{i}+1}^{N_{\tilde{m}}^{i}} Z_{k} / \sigma_{i, 0} \sqrt{\widetilde{m}}$ almost everywhere converges to 0 as $N \stackrel{k=\tau_{m}^{i}}{N_{m}^{i}+\infty}$; therefore,

$$
\frac{-c_{1}+\tilde{m} \mu_{i, 0}(1+o(1))}{\sigma_{i, 0} \sqrt{\widetilde{m}}}+\frac{\sum_{k=\tau_{N_{\tilde{m}}^{i}}}^{N_{\widetilde{m}}^{i}} Z_{k}}{\sigma_{i, 0} \sqrt{\widetilde{m}}}=\frac{-\underline{\mu}_{0}+\varepsilon_{1} \mu_{i, 0}}{\sigma_{i, 0} \sqrt{\varepsilon_{1}}} \sqrt{N}+o(1) .
$$

Notice that, for a large $x$, the relationship between probability distribution function $\Phi(x)$ and density function $p(x)$ of standard normal distribution is

$$
1-\Phi(x)=\frac{p(x)}{x}(1+o(1))
$$

Combining (16)-(18) and the central limit theorem of Markov chain (see [32]), we can get that

$$
\begin{aligned}
\mathbb{P}_{0}\left(S_{\tilde{m}} \leq c_{1} \mid X_{0}=s_{i}\right) & =1-\Phi\left(\frac{-\underline{\mu}_{0}+\varepsilon_{1} \mu_{i, 0}}{\sigma_{i, 0} \sqrt{\varepsilon_{1}}} \sqrt{N}+o(1)\right)(1+o(1)) \\
& =\frac{\sigma_{i, 0} \sqrt{\varepsilon_{1}}(1+o(1))}{\sqrt{2 \pi N}\left(-\underline{\mu}_{0}+\varepsilon_{1} \mu_{i, 0}\right)} \exp \left[-\frac{\left(-\underline{\mu}_{0}+\varepsilon_{1} \mu_{i, 0}\right)^{2}}{2 \sigma_{i, 0}^{2} \varepsilon_{1}} N\right] \\
& \leq \frac{\bar{\sigma}_{0} \sqrt{\varepsilon_{1}}(1+o(1))}{\sqrt{2 \pi N} \underline{\mu}_{0}\left(\varepsilon_{1}-1\right)} \exp \left[-\frac{\underline{\mu}_{0}^{2}\left(\varepsilon_{1}-1\right)^{2}}{2 \bar{\sigma}_{0}^{2} \varepsilon_{1}} N\right] .
\end{aligned}
$$


Put (19) into (15) and combine $\sum_{i=1}^{\infty} \mathbb{P}_{0}\left(X_{0}=s_{i}\right)=1$ to yield that

$$
\mathbb{P}_{0}\left(T_{Z}\left(c_{1}\right)>\widetilde{m}\right) \leq \frac{\bar{\sigma}_{0} \sqrt{\varepsilon_{1}}(1+o(1))}{\sqrt{2 \pi N} \underline{\mu}_{0}\left(\varepsilon_{1}-1\right)} \exp \left[-\frac{\underline{\mu}_{0}^{2}\left(\varepsilon_{1}-1\right)^{2}}{2 \bar{\sigma}_{0}^{2} \varepsilon_{1}} N\right] .
$$

For $1 \leq m \leq \tilde{m}-1$, by the Markov inequality and $\tilde{m}=\left[\varepsilon_{1} N\right]$, we have

$$
\begin{aligned}
\sum_{m=1}^{\tilde{m}-1} \mathbb{P}_{0}\left(T_{Z}\left(c_{1}\right)>m\right) & =\sum_{m=1}^{\tilde{m}-1} \mathbb{P}_{0}\left(Z_{1} \leq c_{1}, \ldots, \sum_{k=1}^{m} Z_{k} \leq c_{1}\right) \\
& \leq(\tilde{m}-1) \mathbb{P}_{0}\left(Z_{1} \leq c_{1}\right) \\
& \leq \tilde{m} \mathbb{P}_{0}\left(\left|Z_{1}\right| \geq-\mu_{0} N\right) \\
& \leq \frac{\tilde{m} \mathbb{E}_{0, s_{i}}\left|Z_{1}\right|^{2}}{\mu_{0}^{2} N^{2}}=\frac{\varepsilon_{1} \mathbb{E}_{0, s_{i}}\left|Z_{1}\right|^{2}}{\mu_{0}^{2} N} .
\end{aligned}
$$

Inequality (21) implies that the value of $\sum_{m=1}^{\tilde{m}-1} \mathbb{P}_{0}\left(T_{Z}\left(c_{1}\right)>m\right)$ tends to 0 as $N \longrightarrow \infty$. Put $(20)$ and (21) into (14); then, there exists some constant $L$ such that

$$
\mathbb{E}_{0}\left(T_{Z}\left(c_{1}\right)\right) \leq 1+\frac{(N-\widetilde{m}+1) \bar{\sigma}_{0} \sqrt{\varepsilon_{1}}(1+o(1))}{\sqrt{2 \pi N} \underline{\mu}_{0}\left(\varepsilon_{1}-1\right)} \exp \left[-\frac{\underline{\mu}_{0}^{2}\left(\varepsilon_{1}-1\right)^{2}}{2 \bar{\sigma}_{0}^{2} \varepsilon_{1}} N\right]<L,
$$

for a large $N$.

Using Theorem 5.1.7 in [33], there exists a nonpositive number $c_{2}=c_{2}(N)>\left(c_{1}(N)\right)$ such that

$$
\mathbb{E}_{0}\left(T_{Z}\left(c_{2}\right)\right)>L
$$

holds for a large enough $N$. Thus, we can choose a negative number $c^{*}=c^{*}(N)$ satisfying $c_{1}<c^{*}<c_{2}$ and $\mathbb{E}_{0}\left(T_{Z}\right.$ $\left.\left(c^{*}\right)\right)=L$.

Next, we prove (10). For any $x>0$, let $c_{3}=-\left(\left|\bar{\mu}_{0}\right|+x\right) N$. Similar to (20), we can get that

$$
\begin{aligned}
1 & \leq \mathbb{E}_{1}\left(T_{Z}\left(c_{3}\right)\right)=1+\mathbb{P}_{1}\left(T_{Z}\left(c_{3}\right)>m\right) \\
& \leq 1+\sum_{m=1}^{\tilde{m}-1} \mathbb{P}_{1}\left(T_{Z}\left(c_{3}\right)>m\right)+(N-\tilde{m}+1) \sum_{i=1}^{\infty} \mathbb{P}_{1}\left(X_{0}=s_{i}\right) \mathbb{P}_{1}\left(s_{\tilde{m}} \leq c_{3} \mid X_{0}=s_{i}\right) \\
& =1+o(1)+(1+o(1))(N-\tilde{m}+1) \Phi\left(-\frac{\left(\left|\underline{\mu}_{0}\right|+x\right) N+\tilde{m} \mu_{i, 1}}{\sigma_{i, 0} \sqrt{\tilde{m}}}\right) \\
& =1+o(1)+\frac{(1+o(1))(N-\tilde{m}+1) \sigma_{i, 1} \sqrt{\tilde{m}}}{\left(\left|\underline{\mu}_{0}\right|+x\right) N+\tilde{m} \mu_{i, 1}} \exp \left[-\frac{\left.\left(\left(\left|\underline{\mu}_{0}\right|+x\right) N+\tilde{m} \mu_{i, 1}\right)^{2}\right]}{2 \sigma_{i, 1}^{1} \tilde{m}}\right] \\
& =1+o(1)+\frac{(1+o(1))\left(1-\varepsilon_{1}\right) \bar{\sigma}_{1} \sqrt{\varepsilon_{1} N}}{\left[\left(\left|\underline{\mu}_{0}\right|+x\right)+\varepsilon_{1} \underline{\mu}_{1}\right] \sqrt{2 \pi}} \exp \left[-\frac{\left(\left(\left|\underline{\mu}_{0}\right|+x\right)+\varepsilon_{1} \underline{\mu}_{1}\right)^{2}}{2 \bar{\sigma}_{1}^{2}} N\right] .
\end{aligned}
$$


Note that the second and third terms on the right-hand side of the last inequality in (24) tends to 0 as $N$ goes to infinity.

This completes the proof.

\section{Estimation of $\mathbf{A R L}_{1}$ with a Positive Control Limit}

In the subsequent discussion, we use the equivalence between limit distribution of a sum and that of a suprema sum of Markov chain to estimate the out-of-control $\mathrm{ARL}_{1}$ when the control limit $c$ is a sufficiently large positive constant. The main result is presented in the following theorem.

Theorem 2. Assume that $\left\{X_{k}, k \geq 0\right\}$ is a homogeneous Markov chain. For any state $s_{i}, i=1,2, \ldots$ if

$$
\mathbb{E}_{1}\left(\eta_{1}^{i}\right)^{2} \leq \overline{\widetilde{\mu}}_{1}^{2}+\overline{\widetilde{\sigma}}_{1}^{2}<\infty,
$$

then

$$
\begin{gathered}
\frac{c-\sqrt{c \ln c}}{\bar{\mu}_{1}}+\frac{(1+o(1)) \underline{\sigma}_{1}}{\varepsilon_{2} \sqrt{2 \pi \bar{\mu}_{1} \ln c}} \exp \left(-\frac{\bar{\mu}_{1}(c-\sqrt{c \ln c})}{2 \underline{\sigma}_{1}^{2}}\right) \leq \mathbb{E}_{1}\left(T_{Z}(c)\right) \\
\leq \frac{c+\sqrt{c \ln c}}{\underline{\mu}_{1}}+\frac{\bar{\sigma}_{1}(1+o(1)) \sqrt{c+\sqrt{c \ln c}}}{\varepsilon_{2} \sqrt{2 \pi \underline{\mu}_{1} c \ln c}} \exp \left(-\frac{\underline{\mu}_{1}(\sqrt{c \ln c})}{2 \bar{\sigma}_{1}^{2}}\right),
\end{gathered}
$$

for large $N$ and $c$, where $\bar{\mu}_{1}, \mu_{1}, \bar{\sigma}_{1}, \sigma_{1}$ and $\overline{\widetilde{\mu}}_{1}, \overline{\widetilde{\sigma}}_{1}$ are defined in (5) and (6), respectively. The sign $o(\cdot)$ denotes the infinitesimal of higher order and $\varepsilon_{2}$ : $=\exp \left[\mu_{1}^{2} / 2 \bar{\sigma}_{1}^{2}\right]-1$.

$$
\mathbb{P}_{1}\left(T_{Z}(c)>m\right)=\mathbb{P}_{1}\left(Z_{1} \leq c, Z_{1}+Z_{2} \leq c, \ldots, \sum_{k=1}^{m} Z_{k} \leq c\right)=\mathbb{P}_{1}\left(\max _{1 \leq k \leq m} S_{k} \leq c\right)
$$

The estimation of $\mathbb{P}_{1}\left(T_{Z}(c)>m\right)$ is reduced to the estimation of $\mathbb{P}_{1}\left(\max _{1 \leq k \leq m} S_{k} \leq c\right)$. According to Theorem 2 in [34], we have the following conclusion:

$$
\lim _{m \longrightarrow \infty} \mathbb{P}_{1}\left(\max _{1 \leq k \leq m} S_{k}-\mathbb{E}_{1} S_{m} \leq x \sqrt{m}\right)=\lim _{m \longrightarrow \infty} \mathbb{P}_{1}\left(S_{m}-\mathbb{E}_{1} S_{m} \leq x \sqrt{m}\right) .
$$

Then, we use (28) to calculate the value of $\mathbb{P}_{1}\left(T_{Z}(c)>m\right)$. Let $\widehat{c}=c-\mathbb{E}_{1} S_{m}=x \sqrt{m}$, where $x \in \mathbb{R}$ and

$\mathbb{R}$ denotes the real number field. When $m$ becomes sufficiently large, by (5), (27), and (28), we have

$$
\begin{aligned}
\mathbb{P}_{1}\left(T_{Z}(c)>m\right) & =\mathbb{P}_{1}\left(\max _{1 \leq k \leq m} S_{k} \leq c\right)=\mathbb{P}_{1}\left(S_{m}-\mathbb{E}_{1} S_{m} \leq \widehat{c}\right)(1+o(1)) \\
& =\mathbb{P}_{1}\left(\frac{S_{m}-\mathbb{E}_{1} S_{m}}{\mathbb{D}_{1} S_{m}} \leq \frac{c-\mathbb{E}_{1} S_{m}}{\mathbb{D}_{1} S_{m}}\right)(1+o(1)) \\
& =\Phi\left(\frac{c-m \mu_{i, 1}}{\sqrt{m} \sigma_{i, 1}}\right)(1+o(1)) .
\end{aligned}
$$

Let $\widehat{m}_{1}=(c+\sqrt{c \ln c}) / \mu_{i, 1}$, and we have 


$$
\begin{aligned}
\mathbb{E}_{1}\left(T_{Z}(c)\right) & =\sum_{m=1}^{\infty} \mathbb{P}_{1}\left(T_{Z}(c)>m\right)=\sum_{m=1}^{\left[\widehat{m}_{1}\right]} \mathbb{P}_{1}\left(T_{Z}(c)>m\right)+\sum_{m=\left[\widehat{m}_{1}\right]+1}^{\infty} \mathbb{P}_{1}\left(T_{Z}(c)>m\right) \\
& =\sum_{m=1}^{\left[\hat{m}_{1}\right]} \mathbb{P}_{1}\left(T_{Z}(c)>m\right)+(1+o(1)) \sum_{m=\left[\hat{m}_{1}\right]+1}^{\infty} \Phi\left(\frac{c-m \mu_{i, 1}}{\sqrt{m} \sigma_{i, 1}}\right) \\
& =\sum_{m=1}^{\left[\hat{m}_{1}\right]} \mathbb{P}_{1}\left(T_{Z}(c)>m\right)+(1+o(1)) \sum_{k=1}^{\infty} \Phi\left(\frac{c-\left(\widehat{m}_{1}+k\right) \mu_{i, 1}}{\sigma_{i, 1} \sqrt{\hat{m}_{1}+k}}\right),
\end{aligned}
$$

for the large enough $m$ and $c$. When $c$ goes to infinity, we deal with the second term on the right-hand side of (30) as follows:

$$
\begin{aligned}
\Phi\left(\frac{c-\left(\widehat{m}_{1}+k\right) \mu_{i, 1}}{\sigma_{i, 1} \sqrt{\hat{m}_{1}+k}}\right) & =\Phi\left(\frac{c-\left(\left(c / \mu_{i, 1}\right)+\left(\sqrt{c \ln c} / \mu_{i, 1}\right)+k\right) \mu_{i, 1}}{\left(\sigma_{i, 1}(\sqrt{c+\sqrt{c \ln c}}) / \mu_{i, 1}\right)+k}\right)=\Phi\left(-\frac{\sqrt{\mu_{i, 1} c \ln c}+\mu_{i, 1} \sqrt{\mu_{i, 1} k}}{\sigma_{i, 1} \sqrt{c+\sqrt{c \ln c}+\mu_{i, 1} k}}\right) \\
& =\frac{(1+o(1)) \sigma_{i, 1}}{\sqrt{2 \pi} \mu_{i, 1}} \frac{\sqrt{c+\sqrt{c \ln c}+\mu_{i, 1} k}}{\sqrt{c \ln c}+\mu_{i, 1} k} \exp \left(-\frac{\mu_{i, 1}\left(\sqrt{c \ln c}+\mu_{i, 1} k\right)^{2}}{2 \sigma_{i, 1}^{2}\left(c+\sqrt{c \ln c}+\mu_{i, 1} k\right)}\right) .
\end{aligned}
$$

Note that, for a large $c$, we have

$$
\begin{aligned}
-\frac{\mu_{i, 1}\left(\sqrt{c \ln c}+\mu_{i, 1} k\right)^{2}}{2 \sigma_{i, 1}^{2}\left(c+\sqrt{c \ln c}+\mu_{i, 1} k\right)} & =-\frac{\mu_{i, 1}^{2} k}{2 \sigma_{i, 1}^{2}}+\frac{\mu_{i, 1}^{2} k c-\mu_{i, 1} c \ln c-\mu_{i, 1}^{2} k \sqrt{c \ln c}}{2 \sigma_{i, 1}^{2}\left(c+\sqrt{c \ln c}+\mu_{i, 1} k\right)} \\
& =-\frac{\mu_{i, 1}^{2} k}{2 \sigma_{i, 1}^{2}}-\frac{\mu_{i, 1} \sqrt{c \ln c}}{2 \sigma_{i, 1}^{2}}+\frac{\mu_{i, 1} c}{2 \sigma_{i, 1}^{2}}\left(1-\frac{c}{c+\sqrt{c \ln c}+\mu_{i, 1} k}\right) \\
& =-\frac{\mu_{i, 1}^{2} k}{2 \sigma_{i, 1}^{2}}-\frac{\mu_{i, 1} \sqrt{c \ln c}}{2 \sigma_{i, 1}^{2}}+o(c) .
\end{aligned}
$$

We obtain the right-hand side of (30) as follows:

$$
\begin{aligned}
\sum_{k=1}^{\infty} \Phi\left(\frac{c-\left(\widehat{m}_{1}+k\right) \mu_{i, 1}}{\sigma_{i, 1} \sqrt{\widehat{m}_{1}+k}}\right) & \leq \frac{\sigma_{i, 1}(1+o(1)) \sqrt{c+\sqrt{c \ln c}}}{\sqrt{2 \pi \mu_{i, 1} c \ln c}} \exp \left(-\frac{\mu_{i, 1} \sqrt{c \ln c}}{2 \sigma_{i, 1}^{2}}\right) \sum_{k=1}^{\infty} \exp \left(-\frac{\mu_{i, 1}^{2} k}{2 \sigma_{i, 1}^{2}}\right) \\
& \leq \frac{\sigma_{i, 1}(1+o(1)) \sqrt{c+\sqrt{c \ln c}}}{\varepsilon_{2} \sqrt{2 \pi \mu_{i, 1} c \ln c}} \exp \left(-\frac{\mu_{i, 1} \sqrt{c \ln c}}{2 \sigma_{i, 1}^{2}}\right),
\end{aligned}
$$


as $c$ tends to infinity. Combining (30), (33), and $\mathbb{P}_{1}\left(T_{Z}(c)>m\right) \leq 1$, we obtain

$$
\begin{aligned}
\mathbb{E}_{1}\left(T_{Z}(c)\right) & =\sum_{m=1}^{\left[\hat{m}_{1}\right]} \mathbb{P}_{1}\left(T_{Z}(c)>m\right)+\frac{\sigma_{i, 1}(1+o(1)) \sqrt{c+\sqrt{c \ln c}}}{\varepsilon_{2} \sqrt{2 \pi \mu_{i, 1} c \ln c}} \exp \left(-\frac{\mu_{i, 1} \sqrt{c \ln c}}{2 \sigma_{i, 1}^{2}}\right) \\
& \leq \widehat{m}_{1}+\frac{\bar{\sigma}_{1}(1+o(1)) \sqrt{c+\sqrt{c \ln c}}}{\varepsilon_{2} \sqrt{2 \pi \underline{\mu}_{1} c \ln c}} \exp \left(-\frac{\underline{\mu}_{1} \sqrt{c \ln c}}{2 \bar{\sigma}_{1}^{2}}\right) \\
& \leq \frac{\underline{\mu}_{1}+\sqrt{c \ln c}}{\underline{\sigma}_{1}}+\frac{\bar{\sigma}_{1}(1+o(1)) \sqrt{c+\sqrt{c \ln c}}}{\varepsilon_{2} \sqrt{2 \pi \mu_{1} c \ln c}} \exp \left(\frac{-\underline{\mu}_{1} \sqrt{c \ln c}}{2 \bar{\sigma}_{1}^{2}}\right) .
\end{aligned}
$$

To prove the inequality on the left-hand side of (26), let $\widehat{m}_{2}=(c-\sqrt{c \ln c}) / \mu_{i, 1}$, and $c$ goes to infinity; then, we use (27) to yield that

$$
\begin{aligned}
\mathbb{E}_{1}\left(T_{Z}(c)\right) & =\sum_{m=1}^{\left[\hat{m}_{2}\right]} \mathbb{P}_{1}\left(T_{Z}(c)>m\right)+(1+o(1)) \sum_{k=1}^{\infty} \Phi\left(\frac{c-\left(\widehat{m}_{2}+k\right) \mu_{i, 1}}{\sigma_{i, 1} \sqrt{\widehat{m}_{2}+k}}\right) \\
& =\sum_{m=1}^{\left[\hat{m}_{2}\right]} \mathbb{P}_{1}\left(\frac{\max _{1 \leq k \leq m} S_{k}-m \mu_{i, 1}}{\sigma_{i, 1} \sqrt{m}} \leq \frac{c-m \mu_{i, 1}}{\sigma_{i, 1} \sqrt{m}}\right)+(1+o(1)) \sum_{k=1}^{\infty} \Phi\left(\frac{c-\left(\widehat{m}_{2}+k\right) \mu_{i, 1}}{\sigma_{i, 1} \sqrt{\hat{m}_{2}+k}}\right) \\
& =\sum_{m=1}^{\left[\widehat{m}_{2}\right]} \mathbb{G}_{1}\left(\frac{c-m \mu_{i, 1}}{\sigma_{i, 1} \sqrt{m}}\right)+(1+o(1)) \sum_{k=1}^{\infty} \Phi\left(\frac{c-\left(\widehat{m}_{2}+k\right) \mu_{i, 1}}{\sigma_{i, 1} \sqrt{\widehat{m}_{2}+k}}\right),
\end{aligned}
$$

where $\quad \mathbb{G}_{1}(x):=\mathbb{P}_{1}\left(\left(\max _{1 \leq k \leq m} S_{k}-m \mu_{i, 1} / \sigma_{i, 1} \sqrt{m}\right) \leq x\right)$ is the distribution function and $m=1,2, \ldots,\left[\widehat{m}_{2}\right]$. Similar to the derivation of (33), let $c$ tend to infinity; then, we obtain the estimation of the second term on the right-hand side of (35) as

$$
\sum_{k=1}^{\infty} \Phi\left(\frac{c-\left(\widehat{m}_{2}+k\right) \mu_{i, 1}}{\sigma_{i, 1} \sqrt{\widehat{m}_{2}+k}}\right) \geq \frac{(1+o(1)) \underline{\sigma}_{1}}{\varepsilon_{2} \sqrt{2 \pi \bar{\mu}_{1} c \ln c}} \exp \left(-\frac{\bar{\mu}_{1}(c-\sqrt{c \ln c})}{2 \underline{\sigma}_{1}^{2}}\right)
$$

For the first term on the right-hand side of (35), because the function $\left(c-m \mu_{i, 1} / \sigma_{i, 1} \sqrt{m}\right)$ is monotonically decreasing in the interval $\left[1,\left[\widehat{m}_{2}\right]\right]$ with respect to $m$ and the distribution function $\mathbb{G}_{1}(x)$ is monotonically nondecreasing in $\mathbb{R}$, we have

$$
\mathbb{G}_{1}\left(\frac{c-m \mu_{i, 1}}{\sigma_{i, 1} \sqrt{m}}\right) \geq \mathbb{G}_{1}\left(\frac{c-\widehat{m}_{2} \mu_{i, 1}}{\sigma_{i, 1} \sqrt{\widehat{m}_{2}}}\right)=\mathbb{G}_{1}\left(\frac{\sqrt{\mu_{i, 1} c \ln c}}{\sigma_{i, 1} \sqrt{c-\sqrt{c \ln c}}}\right)=\mathbb{G}_{1}\left(\frac{\sqrt{\mu_{i, 1} \ln c}}{\sigma_{i, 1} \sqrt{1-(\ln c / c)}}\right) \approx 1
$$


TABLE 1: Simulation values of $\mathrm{ARL}_{1}$ for $N=1000$.

\begin{tabular}{lccc}
\hline $\mathrm{ARL}_{0}$ & 250 & 370 & 500 \\
\hline $\mathrm{ARL}_{0}$ & 251 & 371 & 501.1 \\
$c$ & $-1.2760 \times 10^{6}$ & $-1.8869 \times 10^{6}$ & $-2.5488 \times 10^{6}$ \\
$\mathrm{ARL}_{1}$ & 1 & 1 & 1 \\
\hline
\end{tabular}

TABLE 2: Ratios of $\mathbb{E}_{1}\left(T_{Z}(c)\right)$ and $c$ with different choices of $N$.

\begin{tabular}{lccccccc}
\hline$N / 100$ & 1 & 5 & 10 & 50 & 100 & 500 & 1000 \\
\hline $\mathbb{E}_{1}\left(T_{Z}(c)\right) / c$ & 2.3801 & 2.4482 & 2.3827 & 2.3662 & 2.4006 & 2.3853 & 2.3880 \\
\hline
\end{tabular}

for $c \longrightarrow \infty$ and $m \in\left[1,\left[\widehat{m}_{2}\right]\right]$. By (35)-(37), we obtain

$$
\mathbb{E}_{1}\left(T_{Z}(c)\right) \geq \widehat{m}_{2}+\frac{(1+o(1)) \underline{\sigma}_{1}}{\varepsilon_{2} \sqrt{2 \pi \bar{\mu}_{1} c \ln c}} \exp \left(-\frac{\bar{\mu}_{1}(c-\sqrt{c \ln c})}{2 \underline{\sigma}_{1}^{2}}\right)
$$

for a large enough $c$. Combine (34) and (38) to yield (26). This completes the proof.

\section{Numerical Experiment}

In this section, we perform two numerical experiments to verify our theoretical results. In our first numerical experiment, let $\left\{X_{k}, k \geq 0\right\}$ be a sequence of i.i.d. Gaussian random variables with the prechange and the postchange probability densities $\quad p_{0}(x)=1 / \sqrt{2 \pi} \exp \left(-\left(x-\mu_{0}\right)^{2} / 2\right) \quad$ and $p_{1}(x)=1 / \sqrt{2 \pi} \exp \left(-\left(x-\mu_{1}\right)^{2} / 2\right)$. It is assumed that the standard case of a shift in the mean of $\left\{X_{k}, k \geq 0\right\}$ from $\mu_{0}$ to $\mu_{1}$ is considered.

According to the proof of Theorem 1, the initial data is chosen as $\mu_{0}=-1, \mu_{1}=100$, and $N=1000$. Given different values of $\mathrm{ARL}_{0}=250,370,500$, we obtain the corresponding values of control limit $c$ and $\mathrm{ARL}_{1}$, which are listed in Table 1.

These results suggest that the value of $\mathbb{E}_{1}\left(T_{Z}(c)\right)$ is equal to 1 with a negative control limit $c$ and a given $A R L_{0}$, which is predicted in Theorem 1 .

In the second experiment, we choose $c=((\exp (1)-1) / \sqrt{\exp (1)}+1) N \quad$ and $N / 100=1,5,10,50,100,500,1000$. Let $\left\{X_{k}, k \geq 0\right\}$ be a homogeneous Poisson process with the prechange and the postchange parameters $\lambda_{0}$ and $\lambda_{1}$; then, the corresponding prechange and postchange transition probabilities are defined as

$$
\mathbb{P}_{r}\left(X_{k}=j \mid X_{k-1}=i\right)= \begin{cases}\frac{\left(\lambda_{r} \Delta t_{k}\right)^{j-i}}{(j-i) !} \exp \left(-\lambda_{r} t\right), & 0<i \leq j \\ 0, & 0 \leq j<i\end{cases}
$$

where $r=0,1$ and $i$ and $j$ are arbitrary states from the state space $S$ and $\Delta t_{k}=t_{k}-t_{k-1}$ for $k \geq 1$.

The results are obtained and presented in Table 2, which show the ratio of $\mathbb{E}_{1}\left(T_{Z}(c)\right)$ and $c$ equals approximately 2.38 as $N \longrightarrow \infty$. It implies that the value of $\mathbb{E}_{1}\left(T_{Z}(c)\right)$ and the control limit $c$ are the infinite of the same order, which is predicted by Theorem 2 .

\section{Data Availability}

The data used to support the findings of the study are generated by Matlab.

\section{Conflicts of Interest}

The authors declare no conflict of interest.

\section{Acknowledgments}

Yan Zhu was supported by NSFC, Grant no. 11801353.

\section{References}

[1] W. A. Shewhart, Economic Control of Quality of Manufactured Product, Van Nostrand, New York, NY, USA, 1931.

[2] V. Barnett and K. F. Turkman, Statistics for the Environment Publising House, John Wiley \& Sons Inc, New Jersey, USA, 1993.

[3] M. Pettersson, "Monitoring a freshwater fish population: statistical surveillance of biodiversity," Environmetrics, vol. 9, no. 2, pp. 139-150, 1998.

[4] R. D. Fricker, Introduction to Statistical Methods for Biosurveillance Publishing House, Cambridge University Press, Cambridge, UK, 2012.

[5] D. Siegmund, "Change-points: from sequential detection to biology and back," Sequential Analysis, vol. 32, no. 1, pp. 2-14, 2013.

[6] M. Frisén, "Evaluations of methods for statistical surveillance," Statistics in Medicine, vol. 11, no. 11, pp. 1489-1502, 1992.

[7] M. Keshavarz, S. Asadzadeh, and S. T. A. Niaki, "Risk-adjusted frailty-based CUSUM control chart for phase I monitoring of patients' lifetime," Journal of Statistical Computation and Simulation, vol. 91, no. 2, pp. 334-352, 2021.

[8] M. Frisén, "Optimal sequential surveillance for finance, public health, and other areas," Sequential Analysis, vol. 28, no. 3, pp. 310-337, 2009.

[9] D. C. Montgomery, Introduction to Statistical Quality control, John Wiley \& Sons Inc, New York, NY, USA, 6th edition, 2009.

[10] P. Qiu, Introduction to Statistical Process Control Publishing House, Chapman \& Hall/CRC, Boca Raton, FL, USA, 2014.

[11] N. A. Adegoke, A. N. H. Smith, M. J. Anderson, and M. D. M. Pawley, "Mewma charts when parameters are estimated with applications in gene expression and bimetal thermostat monitoring," Journal of Statistical Computation and Simulation, vol. 91, no. 1, pp. 37-57, 2021.

[12] M. Erfanian, B. Sadeghpour Gildeh, and M. Reza Azarpazhooh, "A new approach for monitoring healthcare 
performance using generalized additive profiles," Journal of Statistical Computation and Simulation, vol. 91, no. 1, pp. 167-179, 2021.

[13] S. J. Mirkamali, “An improved exponentially weighted moving average chart for monitoring proportions using maxima nomination sampling," Journal of Statistical Computation and Simulation, vol. 91, no. 2, pp. 282-299, 2021.

[14] C. Sonesson and D. Bock, "A review and discussion of prospective statistical surveillance in public health," Journal of the Royal Statistical Society: Series A (Statistics in Society), vol. 166, no. 1, pp. 5-21, 2003.

[15] Z. Song, A. Mukherjee, and J. Zhang, "Some robust approaches based on copula for monitoring bivariate processes and component-wise assessment," European Journal of Operational Research, vol. 289, no. 1, pp. 177-196, 2021.

[16] G. D. Williamson and G. Weatherby Hudson, "A monitoring system for detecting aberrations in public health surveillance reports," Statistics in Medicine, vol. 18, no. 23, pp. 3283-3298, 1999.

[17] W. H. Woodall, M. J. Zhao, K. Paynabar, R. Sparks, and J. D. Wilson, "An overview and perspective on social network monitoring," IISE Transactions, vol. 49, no. 3, pp. 354-365, 2017.

[18] P. Charongrattanasakul and A. Pongpullponsak, "Minimizing the cost of integrated systems approach to process control and maintenance model by EWMA control chart using genetic algorithm," Expert Systems with Applications, vol. 38, no. 5, pp. 5178-5186, 2011.

[19] A. J. Duncan, "The economic design of $\mid$ barX charts used to maintain current control of a process," Journal of the American Statistical Association, vol. 51, no. 274, pp. 228-242, 1956.

[20] E. M. Saniga, "Economic statistical control-chart designs with an application to X and R charts," Technometrics, vol. 31, no. 3, pp. 313-320, 1989.

[21] W. C. Yeong, M. Chong, L. M. Ha, and M. A. Rahim, "Economically optimal de- sign of a multivariate synthetic $t^{2}$ chart," Communication in Statistics Simulation and Computation, vol. 43, no. 6, pp. 1333-1361, Article ID 731122, 2012.

[22] A. A. Aly, R. M. Hamed, and M. A. Mahmoud, "Optimal design of the adaptive exponentially weighted moving average control chart over a range of mean shifts," Communications in Statistics - Simulation and Computation, vol. 46, no. 2, pp. 890-902, 2017.

[23] W. M. Carlyle, D. C. Montgomery, and G. C. Runger, "Optimization problems and methods in quality control and improvement," Journal of Quality Technology, vol. 32, no. 1, pp. 1-17, 2000.

[24] R. Shokrizadeh, A. Saghaei, and V. Amirzadeh, "Optimal design of the variable sampling size and sampling interval variable dimension T2 control chart for monitoring the mean vector of a multivariate normal process," Communications in Statistics - Simulation and Computation, vol. 47, no. 2, pp. 329-337, 2018.

[25] Y. Wu, "Estimation of common change point and isolation of changed panels after sequential detection," Sequential Analysis, vol. 39, no. 1, pp. 52-64, 2020.

[26] L. Xie, Y. Xie, and G. V. Moustakides, "Sequential subspace change point detection," Sequential Analysis, vol. 39, no. 3, pp. 307-335, 2020.

[27] T. L. Lai, "Information bounds and quick detection of parameter changes in stochastic systems," IEEE Transactions on Information Theory, vol. 44, no. 7, pp. 2917-2929, 1998.
[28] G. Lorden, "Procedures for reacting to a change in distribution," The Annals of Mathematical Statistics, vol. 42, no. 6, pp. 1897-1908, 1971.

[29] A. S. Polunchenko and V. Raghavan, "Comparative performance analysis of the Cumulative sum chart and the shiryaeroberts procedure for detecting changes in autocorrelated data," Applied Stochastic Models in Business and Industry, vol. 34, no. 6, pp. 922-948, 2018.

[30] M. Frisén, "Statistical surveillance optimality and methods," International Statistical Review, vol. 71, no. 2, pp. 403-434, 2003.

[31] D. Han, F. Tsung, and J. Xian, "Optimal sequential tests for monitoring changes in the distribution of finite observation sequences," 2019, https://arxiv.org/abs/1907.13421.

[32] R. N. Bhattacharya and E. C. Waymire, Stochastic Process with Applications Publishing House, Wiley, New York, NY, USA, 1990.

[33] F. Gregory and L. Vlada, Random Walk: A Modern Introduction, Cambridge University Press, Cambridge, England, UK, 2010.

[34] L. Gao and D. Han, "Extreme value distributions for two kinds of path sums of Markov chain," Methodology and Computing in Applied Probability, vol. 22, no. 1, pp. 279-294, 2020.

[35] W. L. Teoh, M. B. C. Khoo, P. Castagliola, and S. Chakraborti, "Optimal design of the double sampling chart with estimated parameters based on median run length," Computers \& Industrial Engineering, vol. 67, pp. 104-115, 2014. 